\title{
Ehrenpromotion
}

Dem Ordinarius für forstliche Bodenkunde an der Universität Göttingen, Professor Dr. WALTER WITTICH, wurde in Anerkennung seiner hervorragenden Forschungsarbeiten über den Wasserhaushalt nordostdeutscher Kiefernwaldböden, die Bedingungen der Streuzersetzung und die Vorgänge der Rohhumusbildung im Walde, sowie auf dem Gebiet der Standortsdiagnose von der Staatswirtschaftlichen Fakultät der Universität München der Doktorgrad ehrenhalber verliehen.

\section{Deutsche forstliche Bibliographie}

Die Deutsche Zentralstelle für forstliche Bibliographie in Freiburg i. Br. hat im Februar 1957 den 19. Jahrgang der deutschen forstlichen Bibliographie herausgebracht. Er umfaßt im wesentlichen die Veröffentlichungen des Jahres 1955 und enthält neben 20 Registerseiten auf 302 Seiten rund 3000 Titel. Die Klassifizierung wurde, wie bereits im vorigen Jahrgang, nach dem Oxford-System vorgenommen.

Es wurde auch diesem Jahrgang wieder ein ausführliches Inhaltsverzeichnis mit den ersten beiden Dezimalstellen des Oxford-Systems vorangestellt; die weitere Unterteilung des Systems kann der von der Deutschen Zentralstelle für forstliche Bibliographie in Freiburg i. Br. gefertigten deutschen Übersetzung des Oxford-Systems entnommen werden, die sich im Druck befindet und in den nächsten Wochen herauskommt.

Die deutsche forstliche Bibliographie erscheint mit dem 19. Jahrgang erstmals in gedruckter Form. Wir entsprechen damit einem häufig geäußerten Wunsch aus Benutzerkreisen, die darüber klagten, daß das bisher verwandte Saugpostpapier zu leicht vergilbt und die einzelnen Bände durch das dicke Papier auch zu sehr anschwellen. Dank dem finanziellen Entgegenkommen des Bundesministeriums für Ennährung, Landwirtschaft und Forsten konnte dieser Mangel nun behoben werden. Leider wäre es zu teuer geworden, die Titel wie bisher einseitig zu drucken. Benutzern, die die Titel ausschneiden und aufkleben wollen, empfehlen wir, ein zweites Exemplar zu erwerben.

Die Zusammenstellung und Veröffentlichung des vorliegenden Jahresbandes wurde von der Deutschen Zentralstelle für forstliche Bibliographie im Auftrag des Bundesministeriums für Ernäbrung, Landwirtschaft und Forsten durchgeführt, das auch dankenswerterweise die Finanzierung der Arbeit übernommen hat.

Die deutsche forstliche Bibliographie 1955 kann zum Preise von 20,- DM je Stück von der Deutschen Zentralstelle für forstliche Bibliographie, Freiburg i. Br., Bertoldstraße 17 , bezogen werden.

AвETZ

\section{BUCHBESPRECHUN GEN}

Abweichung und Berichtigung der Einzelstamm- und Bestandesmessung nach dem
Verfahren „Hohenadl“ gegenïber der Massenermittlung nach 2-m-Sektionen. Von
FrITZ ENGSTLER, 43 S., $10 \mathrm{Abb}$, brosch. 3,50 DM, Verlag Eugen Ulmer, Ludwigs-
burg, Bd. XII, H. $1 \mathrm{~d}$. Mitt. d. Württ. Forstl. Versuchsanstalt.
Beim Aufmessen yon längeren Baumschäften mit dem Meßverfahren von HoHENADI wird
der Inhalt des neiloidisch ausgeformten bodennahen Schaftstickes systematisch zu klein ge-
funden. Auf der Grundlage eines rd. 1200 Probestämme umfassenden Materials untersucht
ENGSTLER diese systematischen Abweichungen und benutzt den gefundenen engen Zusammen-
hang zwischen dieser systematischen Abweichung und dem bekannten HoHENADL-Quotienten 